\title{
Retrospective review of 108 breast reconstructions using the round block technique after breast- conserving surgery: Indications, complications, and outcomes
}

\author{
Seok Kyung $\operatorname{In}^{1}$, Yoon Soo Kim ${ }^{1}$, Ho Sung Kim ${ }^{1}$, Jin Hyung Park ${ }^{1}$, Hong Il Kim ${ }^{1}$, Hyung Suk Yi ${ }^{1}$, \\ Jea Chun Park ${ }^{2}$, Chang Wan Jeon ${ }^{3}$, Jin Hyuk Choi ${ }^{3}$, Sung Ui Jung ${ }^{3}$, Hyo Young Kim ${ }^{1}$ \\ Departments of ${ }^{1}$ Plastic and Reconstructive Surgery, ${ }^{2}$ Radiology, and ${ }^{3}$ Surgery, Kosin University Gospel Hospital, Busan, Korea
}

Background Several oncoplastic approaches have been implemented in recent years to enhance cosmetic results and to reduce complications. The round block technique is a volume displacement technique for breast reconstruction after breast-conserving surgery (BCS). However, its indications are currently limited according to tumor location, and its cosmetic results and complications have not been clearly established. We hypothesized that the round block technique could produce favorable cosmetic results without major complications regardless of tumor location or nipple-tumor distance, below a certain resected tumor volume and tumor-breast volume ratio.

Methods All breast reconstructions using the round block technique after BCS were included in this analysis. Patients' data were reviewed retrospectively to investigate complications during follow-up, and clinical photos were used to evaluate cosmetic results. The relationships of tumor location, nipple-tumor distance, tumor volume, and the tumor-breast volume ratio with cosmetic results were investigated.

Results In total, 108 breasts were reconstructed. The mean resected tumor volume was $30.2 \pm 15.0 \mathrm{~mL}$. The cosmetic score was $4.5 \pm 0.6$ out of 5 . Tumor location, nipple-tumor distance, tumor volume, tumor-breast volume ratio, radiotherapy, and chemotherapy had no significant effects on cosmetic results or complications. There were no major complications requiring reoperation.

Conclusions Breast reconstruction using the round block technique after BCS can lead to good cosmetic results without major complications regardless of the tumor location, nippletumor distance, radiotherapy, or chemotherapy. Below the maximum tumor volume $(79.2 \mathrm{~mL})$ and the maximum tumor-breast volume ratio (14\%), favorable results were consistently obtained.

Keywords Breast reconstruction / Breast-conserving surgery / Surgical flap
Correspondence: Hyo Young Kim Department of Plastic and Reconstructive Surgery, Kosin University Gospel Hospital, Kosin University College of Medicine, 262 Gamcheon-ro, Seo-gu, Busan 49267, Korea

Tel: +82-51-990-6131

Fax: +82-51-990-6312

E-mail: hose3290@naver.com

This study was supported by a grant from Kosin University College of Medicine (Kosin-19-06). 


\section{INTRODUCTION}

Breast-conserving surgery (BCS) with radiation therapy ( $\mathrm{RTx}$ ) and chemotherapy $(\mathrm{CTx})$ is the gold standard for early breast cancer treatment $[1,2]$. Many studies have demonstrated that BCS is comparable to total mastectomy in terms of the local recurrence rate and overall survival $[3,4]$. Furthermore, BCS is superior to total mastectomy in terms of both the efficacy of oncologic treatment and the psychological consequences of treatment [5].

However, complications such as depression, contraction deformity, seroma, hematoma, and infection are relatively common in BCS when a relatively large amount of tissue is excised $[6,7]$. In recent years, several oncoplastic approaches have been implemented to increase the maximum amount of tissue that can be resected, while still achieving favorable cosmetic results and minimizing the risk of complications. In particular, the round block technique, which is a volume displacement technique used for oncoplastic surgery, involves glandular tissue rearrangement. The advantages of this technique include the lack of donor site morbidity and an inconspicuous postoperative scar, which has a periareolar location [8]. Furthermore, the round block technique is generally applicable when the tumor is located superiorly or close to the nipple $[9,10]$, and its complications or outcomes have not been reported in detail.

The aim of this study was to evaluate the usefulness of the round block technique and to review its complications and cosmetic results. We assumed that the round block technique can produce favorable cosmetic results without major complications regardless of tumor location and nipple-tumor distance.

\section{METHODS}

\section{Study design}

A retrospective review of medical records was performed according to institutional guidelines. In total, 108 patients with breast cancer who underwent BCS at the breast surgery department and immediate breast reconstruction using the round block technique from March 2018 to July 2019 were enrolled. All patients underwent surgery with the round block technique by one plastic surgeon (HYK) after partial mastectomy with sentinel lymph node biopsies or with axillary lymph node dissection by a breast surgeon (CWJ). Reconstruction surgery was recommended to patients who were expected to have a deformity after BCS, regardless of tumor location. We recorded the patients' age, body mass index (BMI), history of hypertension and diabetes mellitus, smoking history, CTx, RTx, tumor location, nipple-tumor distance, tumor volume, breast volume, and tumor-breast volume ratio.

\section{Surgical technique}

The preoperative design was marked by the plastic surgeon. The distance between the inner periareolar circle and the outer circle ranged from $0.5 \mathrm{~cm}$ to $1.5 \mathrm{~cm}$ according to tumor size and the nipple-tumor distance. The initial incision was made on the outer circle by the breast surgeon, and a partial mastectomy with

\section{Fig. 1. Representative intraoperative clinical photos}

(A) After breast-conserving surgery, a depression deformity was observed on the upper outer quadrant of the left breast. (B) A glandular advancement flap was elevated from the pectoralis major muscle and approximated with a Vicryl 1-0 suture. (C) A tethering deformity was noted. (D) Cutaneous redraping was performed. De-epithelialization was performed between the outer and inner circles, and a purse-string suture was placed.
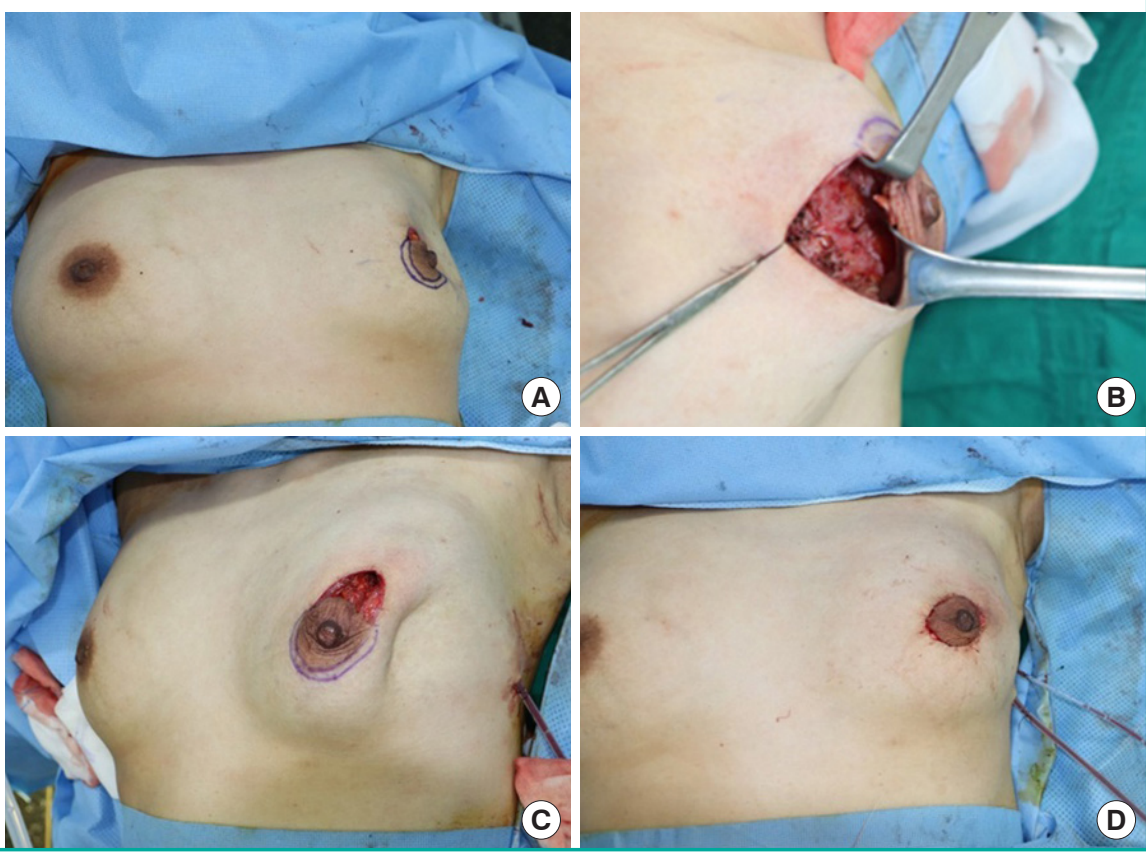
sentinel lymph node biopsies was performed. After partial mastectomy, the plastic surgeon performed reconstruction (Fig. 1A). To cover the defect, a glandular advancement flap was elevated from the pectoralis muscle from both sides according to the tumor location (Fig. 2A and C). Bleeding was controlled and fibrin sealant (TISSEEL; Baxter, Deerfield, IL, USA) was applied. A suction drain was inserted into the sub-glandular area. Glandular flaps were approximated with a Vicryl 1-0 suture (Fig. 1B), and as the glandular flap was moved to the defect site, a tethering deformity was formed (Fig. 1C). In order to eliminate the deformity, cutaneous glandular dissection and cutaneous redraping were performed and a subcutaneous suction drain was inserted (Fig. 2B and D). The marked area between the outer and inner circles was de-epithelialized. A periareolar purse-string suture was placed using Prolene 2-0, and areola tightening was performed following the diameter of the opposite areola (Fig. 1D). A subcuticular dermal running suture was performed with Monosyn 4-0, a skin suture was done with nylon 5-0, and a Steri-Srip (3M, St. Paul, MN, USA) was applied. The mean operative time was $50 \pm 11$ minutes. The resected breast specimens were measured using an electric scale. To measure the breast volume, a beaker was placed on a flat surface and filled with $500 \mathrm{~mL}$ of normal saline. The breast tissue was placed in the beaker, and the increase in the water volume was measured and recorded [11]. A moderate compression dressing with elastic band was maintained and the suction drain was removed when the drainage volume was less than $10-15 \mathrm{~mL}$, generally on postoperative day 4 or 5 .

\section{Follow-up}

Clinical photographs were taken at an outpatient follow-up visit 1 year after surgery. Two plastic surgeons (SKI and YSK) each scored the cosmetic outcomes in terms of three factors (breast shape, breast symmetry, and postoperative scar). Each factor was scored on a 5-point scale, and the scores were averaged for analysis [12]. The effects of age, BMI, tumor location, nippletumor distance, resected tumor volume, tumor-breast volume ratio, CTx, RTx, and diabetes on cosmetic outcomes and complication rates were evaluated.

\section{Preoperative breast volume measurements}

Patients were examined with a $3.0 \mathrm{~T}$ magnetic resonance imaging (MRI) unit (Discovery MR750w 3.0T; GE Healthcare, Milwaukee, WI, USA). Breast cups were used to avoid any compression of the breasts. Non-fat-saturated, T2-weighted axial slice images with a 3-mm thickness were used. All volumetric analyses were conducted using the software's volume rendering algorithm (Fuji Synapse 3D; Fujifilm Medical Systems, Stamford, CT, USA). All measurements were made by one radiologist for consistency. The breast borders were delineated manu-

\section{Fig. 2. Schematic illustration of the surgical procedure}

(A) When the tumor was located above the nipple, a glandular advancement flap was elevated from the pectoralis muscle (dotted blue line). (B) In order to eliminate the tethering deformity, cutaneous glandular dissection was performed (dotted red line). (C) When the tumor was located in the lower outer quadrant, a mainly upper glandular advancement flap was elevated from the pectoralis muscle (dotted blue line). (D) The upper glandular advancement flap was anchored to pectoralis major muscle to prevent inframammary fold retraction. Cutaneous glandular dissection was performed (dotted red line).

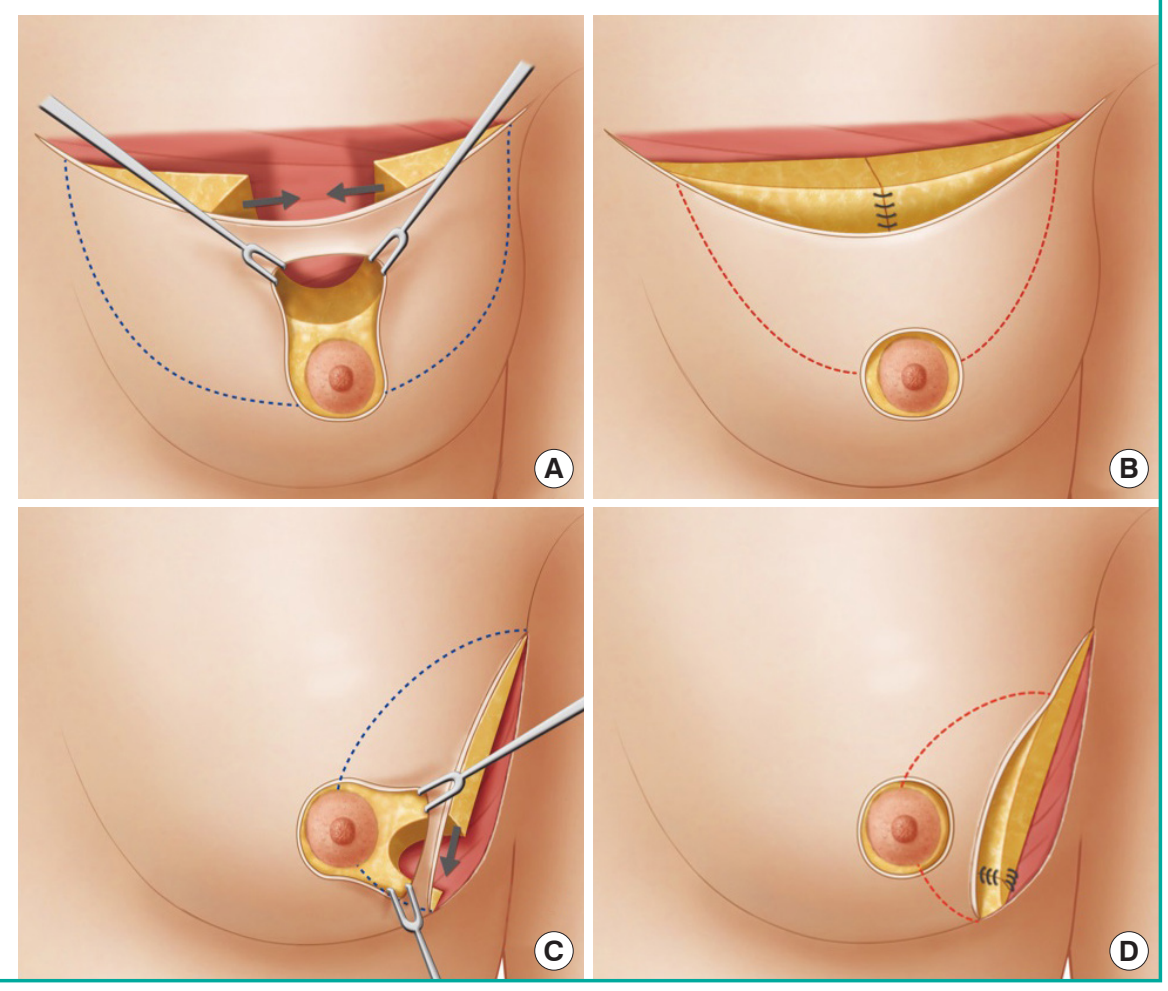


ally by the radiologist on the image showing the highest protrusion. The overall volume was automatically calculated for each breast and was displayed in the software program.

\section{Statistical analysis}

Multiple regression analysis was performed to identify the factors affecting postoperative complications and problematic cosmetic outcomes. For each factor, the Pearson correlation test, the Student t-test or Kruskal-Wallis test, and linear-by-linear association were performed as appropriate. The statistical analysis was conducted using SPSS version 18.0 (SPSS Inc., Chicago, IL, USA). In all statistical comparisons, a P-value of $<0.05$ was considered to indicate statistical significance.

\section{RESULTS}

\section{Patient characteristics}

In total, 108 patients with an mean age of 50.6 years (range, 2568 years) had 108 immediate breast reconstructions using the round block technique following BCS. Of these patients, 55 had tumors located in the upper outer quadrant of the breast, 20 had tumors in the upper inner quadrant, 30 had tumors in the lower outer quadrant, and three had tumors in the lower inner quadrant (Fig. 3). Patients' baseline characteristics and postoperative data are shown in Table 1 . The mean resected tissue volume was $30.2 \mathrm{~mL}$ (range, 4.3-79.2 $\mathrm{mL}$ ), the mean breast volume was 553 $\mathrm{mL}$ (range, 178-997 $\mathrm{mL}$ ), and the mean tumor-breast volume ratio was $5.7 \%$ (range, $1.2 \%-14.7 \%$ ).

\section{Postoperative complication data}

Breast reconstruction with the round block technique had a total complication rate of $11.1 \%$ (108 reconstructions), including seven seromas $(6.5 \%)$, three cases of mild wound dehiscence (2.8\%), and two infections (1.9\%) (Table 2). No complications required a revisional operation. No statistically significant associations were found between the incidence of postoperative complications and CTx, RTx, BMI, diabetes, tumor location, and resected tumor volume, nipple-tumor distance, or the tumor-breast volume ratio.

Table 1. Patients' baseline characteristics and operative data

\begin{tabular}{|c|c|}
\hline Variable & Value \\
\hline Total number of reconstructions & 108 \\
\hline Age (yr) & $50.6 \pm 8.9(25-68)$ \\
\hline Body mass index $\left(\mathrm{kg} / \mathrm{m}^{2}\right)$ & $23.6 \pm 3.2(16.4-36.1)$ \\
\hline Distance from nipple $(\mathrm{cm})$ & $5.5 \pm 1.7(1-10)$ \\
\hline \multicolumn{2}{|l|}{ Breast tumor quadrant } \\
\hline Lower inner & $3(2.8)$ \\
\hline Lower outer & $30(27.8)$ \\
\hline Upper inner & $20(18.5)$ \\
\hline Upper outer & $55(50.9)$ \\
\hline \multicolumn{2}{|l|}{ Histologic type } \\
\hline Invasive ductal carcinoma & $88(81.5)$ \\
\hline Ductal carcinoma in situ & $10(9.3)$ \\
\hline Invasive lobular carcinoma & $4(3.7)$ \\
\hline Mucinous carcinoma & $4(3.7)$ \\
\hline Tubular carcinoma & $1(0.9)$ \\
\hline Medullary carcinoma & $1(0.9)$ \\
\hline Resected breast tumor volume (mL) & $30.2 \pm 15.0(4.3-79.2)$ \\
\hline Tumor-breast volume ratio (\%) & $5.7 \pm 3.0(1.2-14.7)$ \\
\hline Diabetes & $2(1.8)$ \\
\hline Complications & 0 \\
\hline Smoking & 0 \\
\hline Complications & 0 \\
\hline Preoperative chemotherapy & $24(22.2)$ \\
\hline Complications & $3(12.5)$ \\
\hline Postoperative chemotherapy & $70(64.8)$ \\
\hline Complications & $9(12.9)$ \\
\hline Postoperative radiation therapy & $87(80.5)$ \\
\hline Complications & $10(11.5)$ \\
\hline Operation time (min) & $50 \pm 11$ \\
\hline \multicolumn{2}{|l|}{ Breast ptosis proportiona) } \\
\hline Normal & $62(57.4)$ \\
\hline Minimal & $28(25.9)$ \\
\hline Moderate & $11(10.2)$ \\
\hline Severe & 7 (6.5) \\
\hline $\begin{array}{l}\text { Opposite-side mastopexy with the round block } \\
\text { technique }\end{array}$ & $3(2.7)$ \\
\hline
\end{tabular}

\section{Fig. 3. Representative clinical photo}

Representative clinical photo with tumor located in the lower inner quadrant. (A) Preoperative clinical photo. (B) Six months postoperative clinical photo.
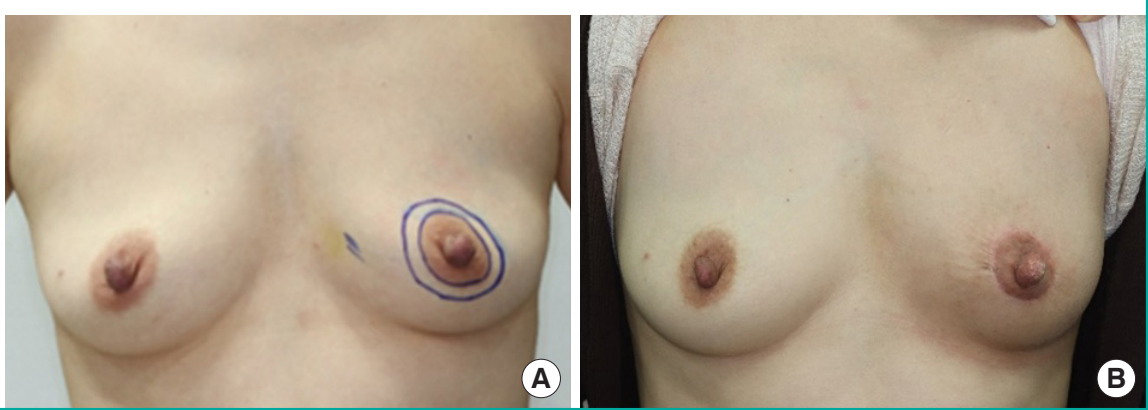
Table 2. Postoperative complications and cosmetic problems

\begin{tabular}{|lc|}
\hline Variable & No. (\%) \\
\hline Total number of reconstructions & 108 \\
Postoperative complications & $12(11.1)$ \\
Seroma & $7(6.5)$ \\
Dehiscence & $3(2.8)$ \\
Infection & $2(1.9)$ \\
Cosmetic problems & $17(15.7)$ \\
Major depression ${ }^{\text {a) }}$ & $2(1.9)$ \\
Minor depression ${ }^{\text {b) }}$ & $6(5.5)$ \\
Bulging deformity & $1(0.9)$ \\
Areola irregularity & $2(1.9)$ \\
Hypopigmented scar & $3(2.8)$ \\
Areolar widening & $3(2.8)$ \\
\hline a)Major depression: depression remained present at 1 year postoperatively; ${ }^{\text {b) Minor }}$ \\
depression: depression had disappeared by 1 year postoperatively. \\
\hline
\end{tabular}

\section{Cosmetic evaluation}

The evaluation of cosmetic outcomes using clinical photographs yielded an mean score of $4.5 \pm 0.6$ points, with no significant differences according to nipple-tumor distance $(\mathrm{P}=0.209)$ or quadrant $(\mathrm{P}=0.267)$ were not statistically significant (Tables 3 , 4). The cosmetic scores tended to worsen as the amount of resection increased, although this trend was not statistically significant $(\mathrm{P}=0.424)$. The tumor-breast volume ratio was also correlated with postoperative cosmetic results, but did not show statistical significance $(\mathrm{P}=0.643)$. $\mathrm{RTx}(\mathrm{P}=0.304)$, and $\mathrm{CTx}$ $(\mathrm{P}=0.225)$ likewise did not show significant relationships with the cosmetic outcomes. We found two major depression deformities (1.9\%), six minor depression deformities (5.5\%), three cases of areolar widening (2.8\%), one immediate bulging deformity ( $0.9 \%)$, three hypopigmented scars $(2.8 \%)$, and two areola irregularities (1.9\%) (Fig. 4). However, over the course of follow-up, the depression deformities and the bulging deformity appeared to resolve, and the 108 patients (with the exception of one who had a major depression), showed satisfactory progression at a 1-year follow-up. In patients with ptotic breasts, asymmetry may occur after the unilateral round block technique. Therefore, in three cases, the same technique was performed on the contralateral side because asymmetry was expected to occur. Breast ptosis was not significantly correlated with the cosmetic score $(\mathrm{P}=0.501)$.

\section{Complication and cosmetic problem rates according to radiation therapy}

In total, 87 patients received RTx after surgery ( $80.5 \%)$. The patients who underwent RTx after surgery had a total complication rate of $11.5 \%$ (10 out of 87 ) and had a total cosmetic problem rate of $16.0 \%$ ( 14 of 87 ). The patients who did not receive
Table 3. Cosmetic score according to tumor location

\begin{tabular}{lrccc}
\hline \multirow{2}{*}{ Tumor location } & \multirow{2}{*}{ No. } & \multicolumn{2}{c}{ Cosmetic score } & \multirow{2}{*}{ P-value } \\
\cline { 3 - 4 } & & Mean \pm SD & Range & \\
\hline Lower inner quadrant & 3 & $4.6 \pm 0.7$ & $3.8-5.0$ & \\
Lower outer quadrant & 30 & $4.7 \pm 0.6$ & $2.9-5.0$ & \\
Upper inner quadrant & 20 & $4.7 \pm 0.4$ & $3.9-5.0$ & \\
Upper outer quadrant & 55 & $4.4 \pm 0.4$ & $2.1-5.0$ & \\
Total & 108 & $4.5 \pm 0.6$ & $2.1-5.0$ & 0.267 \\
\hline
\end{tabular}

Objective analysis of the cosmetic results of patients treated with the round block technique by two plastic surgeons. Mean \pm SD was scored on a 5-point scale. akruskal-Wallis test.

Table 4. Statistical analysis of cosmetic scores according to patient-related factors

\begin{tabular}{|c|c|c|}
\hline Factor & Value & P-value \\
\hline Nipple-tumor distance (cm) & $5.5 \pm 1.7$ & $0.209^{\mathrm{a})}$ \\
\hline Tumor volume (mL) & $30.2 \pm 15.0$ & $0.424^{a)}$ \\
\hline Tumor-breast volume ratio (\%) & $5.7 \pm 3.0$ & $0.643^{\mathrm{a})}$ \\
\hline Radiation therapy & 80.5 & $0.304^{b)}$ \\
\hline Chemotherapy & 64.8 & $0.225^{b)}$ \\
\hline
\end{tabular}

RTx after surgery had a total complication rate of 9\% (2 of 21) and had a total cosmetic problem rate of $14.3 \%$ (3 of 21 ). The odds ratios of RTx for having complications or cosmetic problems were 1.23 and 1.15 , respectively, and these relationships were not statistically significant $(\mathrm{P}=0.576, \mathrm{P}=0.570$, respectively) (Table 5).

\section{DISCUSSION}

Regardless of tumor location, the nipple-tumor distance, tumor volume, and tumor-breast volume ratio, breast reconstruction using the round block technique is a good option for breast cancer patients who undergo partial mastectomy. In our study, the mean tumor-breast volume ratio was 5.7\% (range, 1.2\%$14.7 \%)$. Because the breast surgeon was consulted for oncoplastic surgery even in cases where the extent of tumor resection was not expected to be large, the tumor-breast volume ratio did not affect the cosmetic outcomes. Other studies have reported that the round block technique was indicated in cases with a resection amount of less than $100 \mathrm{~g}$ or a tumor-breast volume ratio that is less than $20 \%$ [13]. However, when the volume is 100 $\mathrm{g}$, the volume excised can be estimated to be $93.45 \mathrm{~mL}[14,15]$. In the authors' experience, reconstruction is challenging in cases where a volume of $93 \mathrm{~mL}$ has been excised if the breast is small. According to prior study, it has been reported that defects corre- 


\section{Fig. 4. Representative clinical photos with mild cosmetic problems}

(A) Bulging deformity (arrow). (B) Depression deformity (arrow). (C) Areolar widening (arrow). (D) Hypopigmented scar (arrow).

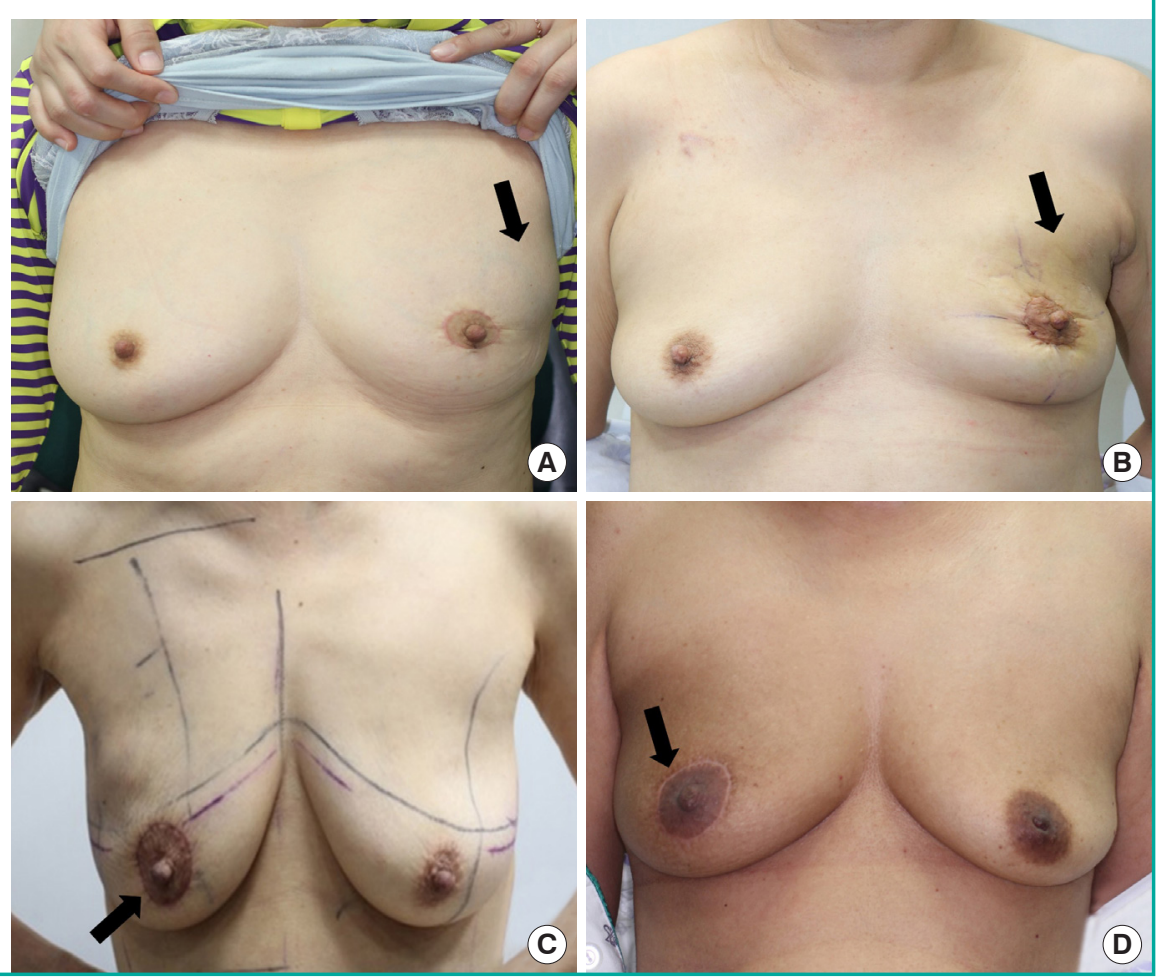

Table 5. Rates of complications and cosmetic problems according to radiation therapy

\begin{tabular}{|c|c|c|c|c|}
\hline & \multicolumn{2}{|c|}{ Radiation therapy } & \multirow{2}{*}{ Odds ratio } & \multirow{2}{*}{ P-value } \\
\hline & Yes & No & & \\
\hline Complications $\mathrm{s}^{\mathrm{a}}$ & & & 1.23 & 0.576 \\
\hline Yes & 10 & 2 & & \\
\hline No & 77 & 19 & & \\
\hline Cosmetic problems $s^{b)}$ & & & 1.15 & 0.570 \\
\hline Yes & 14 & 3 & & \\
\hline No & 73 & 18 & & \\
\hline
\end{tabular}

${ }^{a}$ Complications: seroma, dehiscence, infection; ${ }^{b}$ Cosmetic problems: major depression, minor depression, bulging deformity, areola irregularity, hypopigmented scar, areolar widening.

sponding to less than $20 \%$ of the volume of the breast do not require oncoplastic surgery, while larger defects (more than 20\% require reconstruction) [13]. The breast volume of Asian patients tends to be small, necessitating reconstruction to achieve good cosmetic results even for defects that correspond to less than $20 \%$ of the breast volume. The largest volume found among the patients in this study was $79 \mathrm{~mL}$, and the largest ratio was $14 \%$. For defects of this size and smaller, reconstruction can produce good results. However, further research is needed because the tumor volume or tumor-breast volume ratio at which the round block technique can yield good results may vary depending on the tumor location.
The round block technique is a volume displacement technique that can be used in the superior region of the nipple-areolar complex (NAC) [16]. However, in our study, roughly $30 \%$ of patients had tumors in the lower inner and lower outer quadrants; this location is suboptimal compared to the superior region, but acceptable results were still achieved. The round block technique has been performed in all quadrants with a modified technique, adding subcutaneous dissection of the entire breast to the conventional round block technique [17]. We did not use the modified technique because of its limitation in terms of the surgical field and the possibility of NAC necrosis when the base of the NAC is excised in cases where the tumor is located close to the NAC.

If the tumor is located in the lower outer quadrant or below the nipple, the level of the inframammary fold may change. The expected outcome should be explained to the patient. To address this issue, we fixed the superior glandular tissue to the inferior chest wall in patients with inferior tumors; the usefulness of this technique will be reported later.

It has been demonstrated that the round block technique can be used when the nipple-tumor distance is close, while the tennis racket method is useful when it is far $[18,19]$. The authors found that the round block technique could be applied even when the nipple-tumor distance was $10 \mathrm{~cm}$. Generally speaking, surgery was possible when the distance between the inner peri- 
areolar circle and the outer circle was less than $1 \mathrm{~cm}$, and surgery was possible for the entire breast with a distance of $1.5 \mathrm{~cm}$.

There were several complications after surgery, but no complications necessitated reoperation. The two patients with major depressions had a tumor volume and tumor-breast volume ratio of $35 \mathrm{~mL}$ and $7.5 \%$, and $43 \mathrm{~mL}$ and $9.2 \%$, respectively.

When the tumor was located close to the skin, the skin flap remained thin; therefore, a depression remained after glandular tissue approximation. However, in most cases, these depressions improved noticeably after 3-4 months. We speculate that this occurred due to the repositioning of repaired glandular tissue scarring and fat tissue, but further study is required. Three patients were concerned about wrinkling of the periareolar wound, which may have been caused by the purse-string suture, although we tried to minimize the area of the de-epithelialization; however, this issue also resolved during follow-up. In a previous study, a subcuticular suture was performed with no purse-string when the inter-circular distance was less than $1 \mathrm{~cm}$ [8]. In the absence of the purse string, we observed clinically noticeable areola widening immediately after performing the suture; therefore, we performed a purse-string suture to match the size of the areola on the other side.

There were two surgical site infections in the current study, both of which were completely treated by first-generation cephalosporin (cefazedone, $2 \mathrm{~g} /$ day) for 1 week. Preoperative prophylactic antibiotics were administered and postoperative antibiotics were not used routinely. There were eight seromas in the current study population. A seroma usually develops in the subcutaneous plane, and seroma formation is prevented by wearing a compression dressing for 1 week and suction drainage inserted into both the sub-glandular area and supra-glandular space. When the suction drainage was only placed in the sub-glandular space, four seromas occurred (24\%). When suction drainage was inserted into both the supra-glandular space and sub-glandular space, two seromas formed (1.8\%); although this procedure appears to be effective, further research involving a larger population is necessary to determine whether this difference is statistically significant. The incidence of seroma is not related to age, CTx, or RTx [20].

Fat grafting after partial mastectomy does not increase the recurrence of breast cancer $[21,22]$. However, in our cases, the majority of the depressions improved naturally. Delayed fat grafts can be performed in patients with persistent depressions, but none of our patients wanted fat grafts.

There have been reports of reconstruction using implants after partial mastectomy. However, since partial mastectomy is often followed by RTx, complications such as capsular contraction are more likely to occur $[23,24]$. Therefore, we did not perform im- plant-based reconstruction.

A limitation of this study is that the ratio of the patient's original breast volume to the excised tissue could have been inaccurate because breast volume was measured using preoperative MRI, while the excised tissue was obtained using the water displacement technique. In particular, breast volume measurements made using MRI tend to be larger than the actual volume $[14,15]$. Therefore, the actual tumor-breast volume ratio is therefore likely to have been greater than our measurements. The relatively short follow-up time (1 year) may be another limitation of this study. Over the course of a longer follow-up period, the probability of retraction deformity of the breast increases.

In addition, when a tumor is located in the lower inner or lower outer quadrant, even if the tumor is small, it is difficult to apply the round block technique if the tumor-breast volume ratio is high. A lower tumor-breast volume ratio is required to produce good results for tumors located lower than the NAC than for those located above the NAC. Additional studies should be conducted to determine the minimum size or ratio at which deformation can occur. Depending on the location of the tumor, detailed indications in terms of the tumor volume and tumorbreast volume ratio are needed in the future.

In conclusion, the round block technique following BCS can be performed for patients with tumors in all quadrants of the breast, yielding favorable cosmetic outcomes. Below the maximum tumor volume $(79.2 \mathrm{~mL})$ and the maximum tumor-breast volume ratio (14.7\%), favorable results were obtained regardless of the resected tumor location, volume, nipple-tumor distance, and tumor-breast volume ratio, and no major complications occurred.

\section{NOTES}

\section{Conflict of interest}

No potential conflict of interest relevant to this article was reported.

\section{Ethical approval}

The study was approved by the Institutional Review Board of Kosin University Gospel Hospital (IRB No. 2020-01-014) and performed in accordance with the principles of the Declaration of Helsinki. Written informed consents were obtained.

\section{Patient consent}

The patients provided written informed consent for the publication and the use of their images. 


\section{Author contribution}

Conceptualization: HY Kim, SK In. Data curation: CW Jeon, JH Choi, SU Jung. Formal analysis: HS Kim, JC Park. Methodology: HS Yi, HI Kim. Project administration: HY Kim, JH Park. Writing original draft: SK In. Writing - review and editing: YS Kim. Approval of final manuscript: all authors.

\section{ORCID}

Seok Kyung In https://orcid.org/0000-0003-4346-7949 Yoon Soo Kim Ho Sung Kim Jin Hyung Park Hong Il Kim Hyung Suk Yi Jea Chun Park Chang Wan Jeon Jin Hyuk Choi Sung Ui Jung Hyo Young Kim https://orcid.org/0000-0002-8073-5511 https://orcid.org/0000-0002-6030-3199 https://orcid.org/0000-0001-9415-2467 https://orcid.org/0000-0002-5505-9945 https://orcid.org/0000-0002-9584-8201 https://orcid.org/0000-0003-1870-1510 https://orcid.org/0000-0003-4312-521X https://orcid.org/0000-0001-5625-0912 https://orcid.org/0000-0002-0131-8593 https://orcid.org/0000-0002-1834-5766

\section{REFERENCES}

1. Fisher B, Anderson S, Bryant J, et al. Twenty-year follow-up of a randomized trial comparing total mastectomy, lumpectomy, and lumpectomy plus irradiation for the treatment of invasive breast cancer. N Engl J Med 2002;347:1233-41.

2. Song WJ, Kang SG, Kim EK, et al. Current status of and trends in post-mastectomy breast reconstruction in Korea. Arch Plast Surg 2020;47:118-25.

3. Veronesi U, Cascinelli N, Mariani L, et al. Twenty-year follow-up of a randomized study comparing breast-conserving surgery with radical mastectomy for early breast cancer. $\mathrm{N}$ Engl J Med 2002;347:1227-32.

4. Fisher B, Anderson S, Redmond CK, et al. Reanalysis and results after 12 years of follow-up in a randomized clinical trial comparing total mastectomy with lumpectomy with or without irradiation in the treatment of breast cancer. N Engl J Med 1995;333:1456-61.

5. Litiere S, Werutsky G, Fentiman IS, et al. Breast conserving therapy versus mastectomy for stage I-II breast cancer: 20 year follow-up of the EORTC 10801 phase 3 randomised trial. Lancet Oncol 2012;13:412-9.

6. Brar P, Jain S, Singh I. Complications of axillary lymph node dissection in treatment of early breast cancer: a comparison of MRM and BCS. Indian J Surg Oncol 2011;2:126-32.

7. Hong KY, Son Y, Chang H, et al. Trends in breast reconstruction: implications for the National Health Insurance Service. Arch Plast Surg 2018;45:239-45.

8. Kim MK, Kim J, Jung SP, et al. Round block technique without cerclage in breast-conserving surgery. Ann Surg Oncol 2013;20:3341-7.

9. Yang JD, Lee JW, Kim WW, et al. Oncoplastic surgical techniques for personalized breast conserving surgery in breast cancer patient with small to moderate sized breast. J Breast Cancer 2011;14:253-61.

10. Kim JB, Kim DK, Lee JW, et al. The usefulness of pedicled perforator flap in partial breast reconstruction after breast conserving surgery in Korean women. Arch Plast Surg 2018; 45:29-36.

11. Yi HS, In SK, Kim HS, et al. Factors affecting mastectomy specimen density in direct-to-implant breast reconstruction. Arch Aesthetic Plast Surg 2019;25:137-41.

12. Kim S, Lee S, Lee $\mathrm{H}$, et al. The safety and cosmetic effect of immediate latissimus dorsi flap reconstruction after breast conserving surgery. J Breast Cancer 2009;12:186-92.

13. Honart JF, Reguesse AS, Struk S, et al. Indications and controversies in partial mastectomy defect reconstruction. Clin Plast Surg 2018;45:33-45.

14. Lee WY, Kim MJ, Lew DH, et al. Three-dimensional surface imaging is an effective tool for measuring breast volume: a validation study. Arch Plast Surg 2016;43:430-7.

15. Ogunleye AA, Deptula PL, Inchauste SM, et al. The utility of three-dimensional models in complex microsurgical reconstruction. Arch Plast Surg 2020;47:428-34.

16. Yang JD, Lee JW, Cho YK, et al. Surgical techniques for personalized oncoplastic surgery in breast cancer patients with small- to moderate-sized breasts (part 2): volume replacement.J Breast Cancer 2012;15:7-14.

17. Refaat M, Abouelnagah G, Awad AT, et al. Modified round block technique for peripherally located early cancer breast, a technique that fits for all quadrants. Breast J 2020;26:4149.

18. Haloua MH, Krekel NM, Winters HA, et al. A systematic review of oncoplastic breast-conserving surgery: current weaknesses and future prospects. Ann Surg 2013;257:60920.

19. Gainer SM, Lucci A. Oncoplastics: techniques for reconstruction of partial breast defects based on tumor location.J Surg Oncol 2011;103:341-7.

20. Gdalevitch P, Ho A, Genoway K, et al. Direct-to-implant single-stage immediate breast reconstruction with acellular dermal matrix: predictors of failure. Plast Reconstr Surg 2014;133:738e-747e.

21. Molto Garcia R, Gonzalez Alonso V, Villaverde Domenech ME. Fat grafting in immediate breast reconstruction: avoiding breast sequelae. Breast Cancer 2016;23:134-40. 
22. Salgarello M, Visconti G, Rusciani A. Breast fat grafting with platelet-rich plasma: a comparative clinical study and current state of the art. Plast Reconstr Surg 2011;127:2176-85.

23. Salzberg CA, Ashikari AY, Koch RM, et al. An 8-year experience of direct-to-implant immediate breast reconstruction using human acellular dermal matrix (AlloDerm). Plast Re- constr Surg 2011;127:514-24.

24. Colwell AS, Damjanovic B, Zahedi B, et al. Retrospective review of 331 consecutive immediate single-stage implant reconstructions with acellular dermal matrix: indications, complications, trends, and costs. Plast Reconstr Surg 2011; 128:1170-8. 